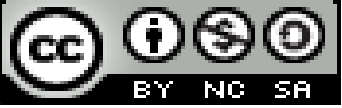

Ciencias de la salud

Artículo Científico

\title{
Efecto anestésico en el bloqueo troncular mandibular, estudio comparativo entre lidocaína $2 \%$ y articaína $4 \%$
}
Anesthetic effect in mandibular nerve block. Comparative study between lidocaine $2 \%$ and articaine $4 \%$

Efeito anestésico no bloqueio troncular mandibular, estudo comparativo entre

\author{
lidocaína $2 \%$ e articaína $4 \%$
}

\author{
Andrés G. Cubero-Flores \\ soyandrescubero@hotmail.com \\ Kleber A. Vallejo-Rosero" \\ avallejo@uce.edu.ec
}

Recibido: 3 de noviembre de 2016 * Corregido: 20 de diciembre de 2016 * Aceptado: 4 de enero 2017

'Odontólogo, Facultad de Odontología, Universidad Central del Ecuador, Quito, Ecuador.

IIDocente, Facultad de Odontología, Universidad Central del Ecuador, Quito, Ecuador. 


\section{Resumen.}

Objetivo: Determinar bajo comparación clínica la eficacia de dos soluciones anestésicas, lidocaína 2\% con epinefrina 1:100000 (Lignospan Standard) y articaína 4\% con epinefrina 1:100000 (Septanest). Materiales y metodos: El tipo de estudio fue experimental in vivo, de campo, comparativa y longitudinal, en el estudio se experimentó con 60 pacientes no comprometidos por enfermedades sistémicas, los cuales fueron sometidos a la enucleación quirúrgica de órganos dentales premolares o molares inferiores: $(3.4,3.5,3.6,3.7,3.8 ; 4.4,4.5,4.6,4.7,4.8)$, como indicación terapéutica, la muestra fue dividida en forma aleatoria en 2 grupos (1 y 2) de 30 individuos cada uno, los datos fueron digitalizados en tablas Excel y sometidos a análisis estadístico mediante el programa SPSS versión 20, se aplicó los test estadísticos Kolmogorov-Smirnov, Shapiro-Wilk y $\mathrm{T}$ student. Resultados: El clorhidrato de articaína 4\% presentó mayores ventajas con respecto al tiempo de latencia, profundidad anestésica y control del dolor comparado clínicamente con el clorhidrato de lidocaína $2 \%$ en la enucleación de premolares o molares inferiores bajo la técnica de anestesia local troncular mandibular método directo. Conclusiones: Se determinó que la articaína 4\% con epinefrina 1:100000 (Septanest), tiene un menor tiempo de latencia en comparación con lidocaína 2\% con epinefrina 1:100000 (Lignospan Standard).

Palabras clave: Anestesia; articaína; lidocaína; bloqueo troncular mandibular. 


\section{Abstract.}

Objective: Determine the efficacy of two anesthetic solutions, lidocaine $2 \%$ with epinephrine 1: 100000 (Lignospan Standard) and 4\% articaine with epinephrine 1: 100000 (Septanest). Materials and Methods: An in vivo, field, comparative and longitudinal study was performed. 60 patients, with no systemic diseases, underwent surgical enucleation of premolars or lower molars: The sample was randomly divided into 2 groups (1 and 2) of 30 individuals each. Data was divided into two groups (1, 2, 3 and 4, 3.4, 3.5, 3.6, 3.7, 3.8, 4.4, 4.5, 4.6, 4.7, 4.8) in Excel tables and subjected to statistical analysis using SPSS version 20 software, Kolmogorov-Smirnov, Shapiro-Wilk and T student statistical tests. Results: Articaine hydrochloride 4\% presented greater advantages regarding latency time, anesthetic depth and pain control, clinically compared to lidocaine hydrochloride $2 \%$, when performing premolars and lower molars enucleation under mandibular block direct method technique. Conclusions: 4\% articaine with epinephrine 1: 100000 (Septanest), has a lower latency time compared to lidocaine 2\% with epinephrine 1: 100000 (Lignospan Standard).

Key words: Anesthesia; Anticaine; Lidocaína; Mandibular Nerve block 


\section{Resumo.}

Objetivo: Determinar mediante comparação clinica, a eficiência de duas soluções anestésicas, lidocaína 2\% com epinefrina 1:100000 (Lignospan Standard) e articaína 4\% com epinefrina 1:100000 (Septanest). Materiais e Métodos: O presente estudo foi experimental in vivo, de campo, comparativo e longitudinal, no qual se experimentou com 60 pacientes sem doenças sistêmicas, os quais foram submetidos a enucleação cirúrgica de órgãos dentários pré-molares ou molares inferiores: $(3.4,3.5,3.6,3.7,3.8 ; 4.4,4.5,4.6,4.7,4.8)$, como indicação terapêutica; a amostra foi dividida aleatoriamente em 2 grupos (1 e 2) de 30 indivíduos cada um, os dados foram digitalizados em tabelas do Excel e submetidas a análise estatístico mediante o programa SPSS versão 20, os testes Kolmogorov-Smirnoc, Shapiro Wilk e t Student foram utilizados. Resultados: Cloridrato de articaína 4\% apresentou maiores benefícios com respeito ao tempo de latência, profundidade anestésica e controle da dor comparado clinicamente com cloridrato de lidocaína $2 \%$ na enucleação de pré-molares e molares inferiores com técnica de anestesia troncular mandibular método direito. Conclusões: Determinou-se que a articaína 4\% com epinefrina 1:100000 (Septanest) tem menor tempo de latência em comparação com a lidocaína 2\% com epinefrina 1:100000 (Lignospan Standard).

Palavras chave: Anestesia, articaína, lidocaína, bloqueio troncular mandibular. 


\section{Introducción.}

El ser humano se encuentra directamente relacionado con el dolor, es así que para evitar estos procesos fisiológicos se buscan nuevas técnicas que permitan la supresión y el control del dolor existiendo una infinidad de elementos, sustancias y plantas a lo largo del tiempo. Sin embargo tras el descubrimiento de las propiedades anestésicas de la planta de coca parte el estudio de las soluciones anestésicas de uso local. En la búsqueda de un anestésico seguro aparecen los primeros anestésicos de tipo amida y entre ellos destaca la lidocaína siendo este anestésico el principal referente de este grupo. (1)

Según Macouzet CO, 2005, (2) la lidocaína es una solución anestésica local tipo amida desarrollado por Nils Lofgren en 1943, además de sus propiedades anestésicas ha sido utilizado como antiarrítmico, presentándose de forma líquida como solución para inyecciones, crema, jalea, aerosol y ungüento.

La lidocaína posee un tiempo de latencia corto, buena profundidad anestésica y su duración es amplia en comparación con otros anestésicos del tipo éster, (3) el clorhidrato de lidocaína está disponible para su uso odontológico en presentaciones al $2 \%$ con o sin vasoconstrictor, el más común epinefrina, en concentraciones 1:80000 y 1:100000. (2)

La articaína, en sus inicios fue conocida con el nombre de carticaína, es un anestésico local de tipo amida que tiene la particularidad de tener en su estructura química un anillo tiofeno que actúa como una partícula lipófila, está disponible en Europa desde 1976, y en otros países como Canadá y Estados Unidos llego después, en 1984 y en el 2000 respectivamente, presentando entre sus características numerosas propiedades similares a las de otros anestésicos, a excepción del anillo

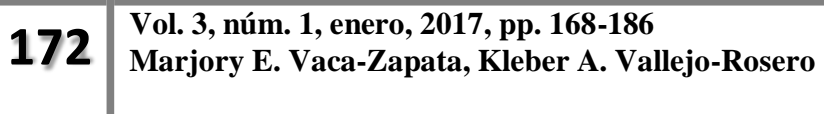


aromático y su gran capacidad de unirse a proteínas. (4) Para Mascouzet CO, (2) el grupo éster que posee la articaína le confiere propiedades menos toxicas que otros anestésicos, ya que este es fácilmente hidrolizado por las proteínas plasmáticas, además la articaína puede difundirse mejor que otros anestésicos en los tejidos duros y blandos.

El clorhidrato de articaína está disponible en formulaciones al 4\% con epinefrina 1:100000 y 1:200000 solución inyectable. (4) Además se encuentran disponible solución de clorhidrato de articaína al $0.5 \%$ con epinefrina 1:200000. (2)

Como todos los anestésicos locales tanto lidocaína como articaína tienen como objetivo el bloqueo reversible de la transmisión nerviosa, disminuyendo la permeabilidad de la membrana de las células nerviosas al ion sodio, este mecanismo suprime toda sensación dolorosa, estimulo de temperatura, tacto, propiocepción y tono de los músculos. (5)

Según Martínez-Martinez AA, 2009, (3) estudios comparativos entre articaína y lidocaína ambos con epinefrina como vasoconstrictor, encontraron eficacia clínica comparable entre los dos anestésicos, otros estudios aseguran una mejor anestesia pulpar a favor de la articaína.

La investigación tuvo como objetivo determinar la eficacia anestésica de la lidocaína al 2\% con epinefrina 1:100000 en comparación con articaína al 4\% 1:100000, de esta manera ayudaremos a encontrar nuevas soluciones anestésicas que brinden seguridad en las acciones clínicas al odontólogo.

El estudio se llevó a cabo en pacientes ambulatorios NO comprometidos con enfermedades sistémicas, de la Clínica de Cirugía de la Facultad de Odontología de la Universidad Central del 
Ecuador, el mismo fue un estudio clínico experimental in vivo, comparativo, de campo y longitudinal entre dos soluciones anestésicas.

Para el presente trabajo de investigación se sustentó el marco teórico con contenidos acerca de neurofisiología, mecanismos de acción de los anestésicos locales, farmacología de ambas soluciones anestésicas, , técnicas de anestesia, instrumentos de medición lo que da veracidad a la investigación.

\section{Materiales y métodos.}

Experimental in vivo, de campo, comparativa y longitudinal.

En el estudio se experimentó con 60 pacientes no comprometidos por enfermedades sistémicas, los cuales fueron sometidos a la enucleación quirúrgica de órganos dentales premolares o molares inferiores: $(3.4,3.5,3.6,3.7,3.8 ; 4.4,4.5,4.6,4.7,4.8)$, como indicación terapéutica, la muestra fue dividida en forma aleatoria en 2 grupos ( 1 y 2 ) de 30 individuos cada uno.

Se realizó la historia clínica de cirugía a cada paciente la cual consta de las siguientes partes: anamnesis, motivo de consulta, enfermedad actual, antecedentes patológicos, examen físico, exámenes complementarios y consentimiento informado, obtuvimos todos los datos que nos permitieron evaluar la condición general de cada paciente y obtener la aprobación de los mismos para formar parte del estudio. Estos documentos médico-legales se encuentran disponibles en el archivo de la Clínica de Cirugía de la Facultad de Odontología de Universidad Central del Ecuador.

Se seleccionaron 30 pacientes de forma aleatoria correspondientes al grupo 1, a los cuales se les administro cartuchos comerciales de anestésico local lidocaína 2\% con epinefrina 1:100000 de 
1,8 ml marca (LIGNOSPAN STANDARD) de la casa comercial Septodont. Se utilizó la técnica de anestesia troncular mandibular método directo para todos los procedimientos, la cual fue realizada por un solo operador que ha culminado la malla curricular de la carrera de Odontología en la Universidad Central del Ecuador, como muestra la $\left(\right.$ Foto $\left.N^{\circ} \mathbf{1}\right)$.

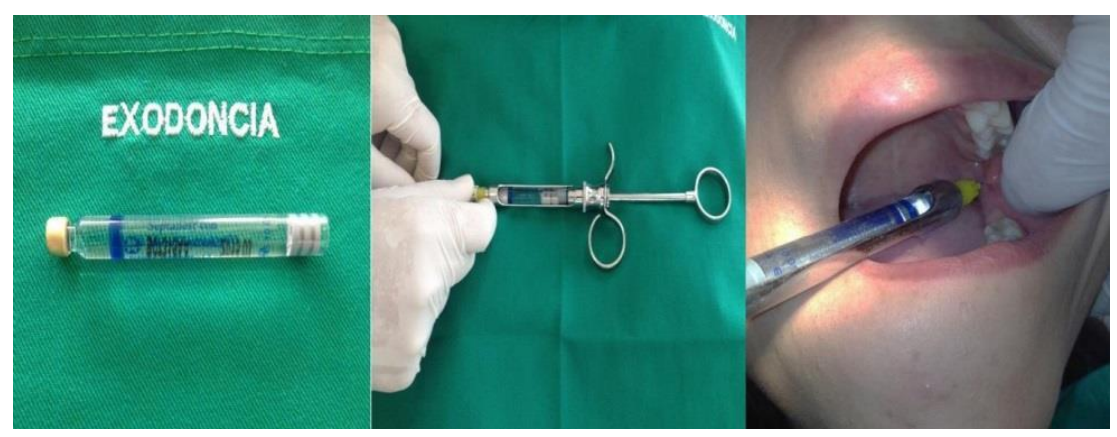

\section{Foto $N^{\circ}$ 1.- Anestesia troncular mandibular método directo con Septanest}

Para la medición del tiempo de latencia se tomó el tiempo transcurrido desde la administración de la solución anestésica, hasta que el anestésico hizo efecto, se realizó la medición del tiempo con cronómetro digital, los datos obtenidos fueron registrados en formularios elaborados por el autor; para este procedimiento se pidió a los pacientes que refieran la aparición de síntomas de anestesia los cuales son: sensación de adormecimiento o frio de la zona anestesiada, comisura labial y punta de la lengua; luego de esto se procedió a verificar la ausencia de estímulos dolorosos de los tejidos duros y blandos de la zona anestesiada, utilizando la parte activa de una pinza de algodón se presionó la encía en varios puntos del cuadrante anestesiado, se pidió a los pacientes que indiquen si la punción causo molestias, como muestra la $\left(\right.$ Foto $\left.N^{\circ} 2\right)$. 


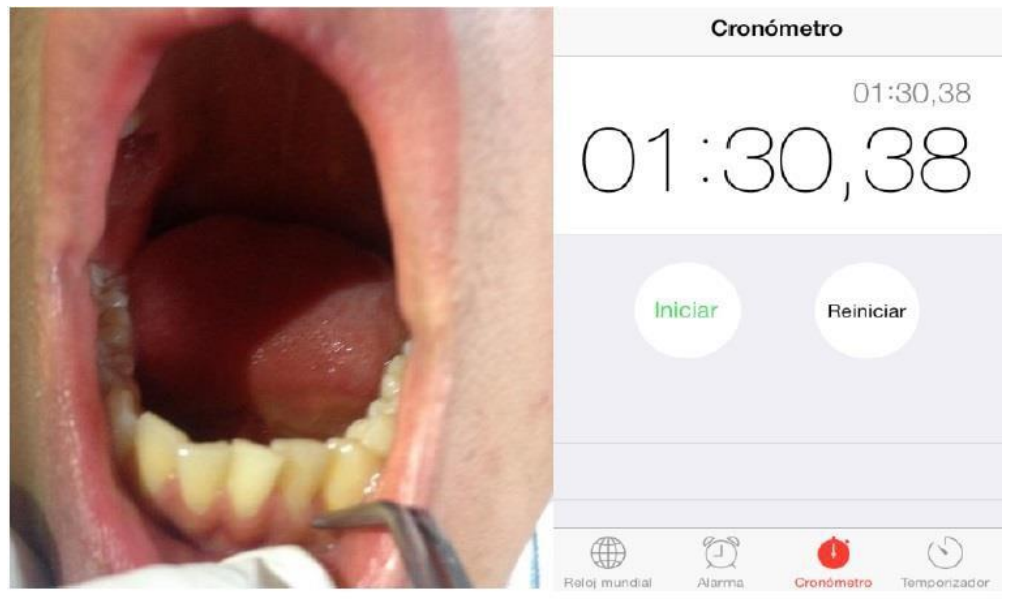

Foto $N^{\circ}$ 2.- Prueba objetiva del tiempo de latencia

Una vez alcanzado el efecto anestésico realizamos la medición de la profundidad anestesica con vitalómetro marca (PULP TESTER DY310 TESTING TEETH NERVE), transcurridos 4 minutos posteriores a la inyección del anestésico para evaluar el nivel de respuesta de los dientes al estímulo eléctrico. Según indicaciones del fabricante no se excedió la potencia de 40 que es la frecuencia eléctrica máxima para evaluar dientes vitales o anestesiados.

Se realizó este procedimiento en un diente con vitalidad del cuadrante anestesiado, previo aislamiento y colocación de pasta profiláctica en una de sus superficies libres, se colocó el electrodo del instrumento y se pidió al paciente que nos señale la percepción de cosquilleo o calor en el diente sometido a la prueba. Los datos obtenidos fueron registrados en formularios elaborados por el autor, como se muestra en la $\left(\right.$ Foto $\left.N^{\circ} 3\right)$. 


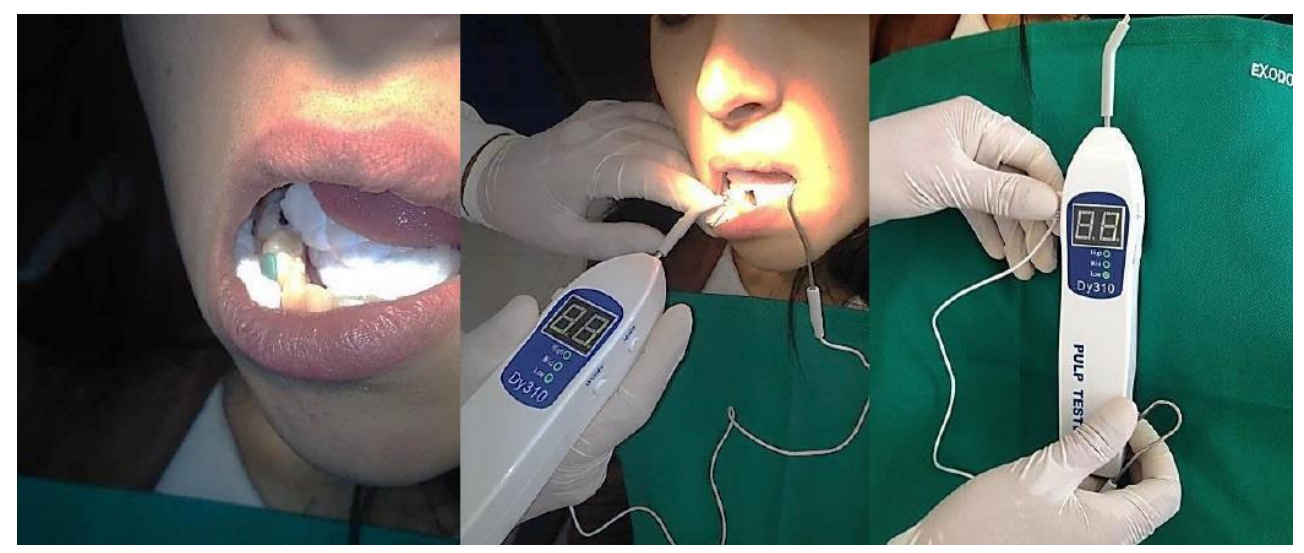

\section{Foto $N^{\circ}$ 3.- Utilización del vitalómetro para evaluar profundidad anestésica}

Al concluir la enucleación del órgano dentario se presentó a los pacientes una Escala Facial del Dolor, la misma consto de 5 niveles: 1. Muy Doloroso, 2. Doloroso, 3. Intermedio, 4. Casi sin Dolor, 5. Ausencia de Dolor. Se pidió a los pacientes que marquen la percepción del dolor que experimentaron durante el procedimiento quirúrgico.

Para la recolección de datos se utilizó un formulario, previamente elaborado por el autor el cual constó de Fecha, Nombre del paciente, Edad, Sexo, Cuadrante \# Diente, Técnica de anestesia, Solución anestésica, Dosis, Prueba con Vitalómetro, Escala de Expresión Facial. Estos formularios fueron digitalizados en tablas Excel y sometidos a análisis estadístico mediante el programa SPSS versión 20 en español, se aplicó los test estadísticos Kolmogorov-Smirnov, Shapiro-Wilk y T student. 


\section{Resultados.}

Determinar mediante comparación clínica el efecto anestésico entre Lidocaína al $2 \%$ y Articaína al 4\% ambas con epinefrina 1:100000 en la técnica de anestesia troncular mandibular método directo.

\begin{tabular}{|c|c|c|c|c|c|c|c|}
\hline \multicolumn{8}{|c|}{ Pruebas de normalidad } \\
\hline & \multirow{2}{*}{ SO LUCIO NES } & \multicolumn{3}{|c|}{ Kolmogorov-Smirnov } & \multicolumn{3}{|c|}{ Shapiro-Wilk } \\
\hline & & Estadístico & Gl & Sig. & Estadístico & Gl & Sig. \\
\hline \multirow{2}{*}{ TIEMPO } & LIDOCAÍNA & 0,113 & 30 & 0,200 & ,962 & 30 & 0,339 \\
\hline & ARTICAÍNA & 0,106 & 30 & 0,200 &, 950 & 30 & 0,168 \\
\hline \multirow{2}{*}{ VITALÓMETRO } & LIDOCAIINA & 0,110 & 30 & 0,200 & ,929 & 30 & 0,047 \\
\hline & ARTICAÍNA & 0,218 & 30 & 0,001 &, 871 & 30 & 0,002 \\
\hline
\end{tabular}

Tabla $N^{\circ}$ 1.- Pruebas de normalidad

\begin{tabular}{cccccc}
\multicolumn{7}{c}{ Estadisticos de grupo } & \\
\hline & & $\mathbf{N}^{\circ}$ & Media & Desviación típ. & Error típ. de la media \\
\hline \multirow{2}{*}{ TIEMPO } & LIDOCAÍNA & 30 & 162,6000 & 12,17572 & 2,22297 \\
& ARTICAÍNA & 30 & 94,3667 & 8,12185 & 1,48284
\end{tabular}

Tabla $N^{\circ}$ 2.- Resultado estadístico de la Prueba T student para tiempo de latencia

\begin{tabular}{ccccc}
\multicolumn{5}{c}{ Rangos } \\
\hline & $\mathbf{N}^{\circ}$ & Rango promedio & Suma de rangos \\
\hline \multirow{2}{*}{ VITALÓMETRO } & LIDOCAÍNA & 30 & 19,60 & 588,0 \\
& ARTICAÍNA & 30 & 41,40 & 1242,00 \\
& TOTAL & 60 & &
\end{tabular}

Tabla $N^{\circ}$ 3.- Resultado estadístico de la Prueba no Paramétrica U Mann-Whitney 
Efecto anestésico en el bloqueo troncular mandibular, estudio comparativo entre lidocaína $2 \%$ y articaína $4 \%$

De la prueba no paramétrica se tiene sig. Asintótica (bilateral) $=0.00<0,05$, luego rechazamos Ho: esto es las muestras provienen de poblaciones similares. En conclusión de la prueba Mann-Whitney la articaína presenta valores MÁS altos por lo que se procede a realizar Prueba t menos estricta por la normalidad.

Estadisticos de grupo

\begin{tabular}{cccccc}
\hline & & $\mathbf{N}^{\circ}$ & Media & Desviacion típ. & Erros típ. de la media \\
\hline \multirow{2}{*}{ VITALÓMETRO } & LIDOCAÍNA & 30 & 19,9000 & 4,07135 & 0,74332 \\
& ARTICAÍNA & 30 & 24,2333 & 4,53099 & 0,82824
\end{tabular}

Tabla $N^{\circ}$ 4.- Resultados estadísticos de la prueba $T$.

Escala Facial del Dolor

\begin{tabular}{|c|c|c|c|c|c|}
\hline & & & \multicolumn{2}{|c|}{ SO LUCIO NES } & \multirow{2}{*}{ Total } \\
\hline & & & LIDO CAINA & ARCAINA & \\
\hline \multirow{8}{*}{ ESCALA_VAS } & \multirow{2}{*}{ DOLOROSO } & Recuento & 5 & 0 & 5 \\
\hline & & $\%$ dentro de SOLUCIONES & 16,7 & 0,0 & 8,3 \\
\hline & \multirow{2}{*}{ INTERMEDIO } & Recuento & 4 & 2 & 6 \\
\hline & & $\%$ dentro de SOLUCIONES & 13,3 & 6,7 & 10,0 \\
\hline & \multirow{2}{*}{ CASI SIN DOLOR } & Recuento & 10 & 8 & 18 \\
\hline & & $\%$ dentro de SOLUCIONES & 33,3 & 26,7 & 30,0 \\
\hline & \multirow{2}{*}{ SIN DOLOR } & Recuento & 11 & 20 & 31 \\
\hline & & $\%$ dentro de SOLUCIONES & 36,7 & 66,7 & 51,7 \\
\hline \multirow{2}{*}{\multicolumn{2}{|c|}{ TOTAL }} & Recuento & 30 & 30 & 60 \\
\hline & & $\%$ dentro de SOLUCIONES & 100,0 & 100,0 & 100,0 \\
\hline
\end{tabular}

\section{Tabla $N^{\circ}$ 5.- Contingencia}

En conclusión el mayor porcentaje de individuos que fueron tratados con Articaína indicaron

“Ausencia de dolor" en un (66,7\%). Las personas tratadas con Lidocaína indican un porcentaje de 
“Ausencia de dolor” (36,7\%), no se tiene un valor representativo como la otra solución, por lo que se afirma que la articaína tiene un mejor control del dolor.

\section{Discusión.-}

El dolor es un proceso fisiológico, que se presenta en la mayoría de pacientes sometidos a procedimientos odontológicos, para evitar esta sensación desagradable en los tratamientos se introdujo en el mercado una amplia gama de elementos, métodos y sustancias utilizadas a lo largo de los años, cada una describe una acción diferente para inhibir el dolor durante los actos clínicos, ya sean estos relacionados con cirugía u otros campos de la odontología donde se realicen procedimientos quirúrgicos cruentos que resultan muy dolorosos para los pacientes. (1)

Los anestésicos locales como la lidocaína y articaína, en sus diferentes presentaciones y concentraciones, tienen como objetivo el bloqueo reversible de la transmisión nerviosa, disminuyendo la permeabilidad de la membrana de las células nerviosas al ion sodio, este mecanismo suprime toda sensación dolorosa, estimulo de temperatura, tacto, propiocepción y tono de los músculos. (5) Al ser un estudio in vivo, se puede determinar una alta confiabilidad de los resultados obtenidos y una gran validez científica, puesto que se utilizó una metodología sistematizada y controlada por los residentes de la especialidad de cirugía oral de la Facultad de Odontología de la U.C.E.

En relación a las soluciones anestésicas utilizadas se escogió cartuchos de anestésico local lidocaína 2\% Lignospan Standard y cartuchos de articaína 4\% Septanest, ambas soluciones pertenecen a la casa comercial francesa Septodont, para tener la seguridad que cumplen con los mismos estándares de elaboración y calidad, estos anestésicos son similares a los utilizados en 
estudios como el de Ensaldo, et al., 2003, (5) en el cual se utilizó lidocaína 2\% Xylocaina y articaína 4\% Medicaina.

Para la conformación del universo de estudio, la muestra estuvo conformada por 60 pacientes sanos sin compromiso de enfermedades sistémicas ni antecedentes alérgicos a los anestésicos locales, a los cuales se les realizo la enucleación de órganos dentales premolares o molares inferiores por motivos ortodonticos, por enfermedades degenerativas de la pulpa o por ser potenciales focos de infección, a 30 pacientes se les administro lidocaína $2 \%$ para el bloqueo mandibular y a los restantes 30 pacientes se les aplico articaína 4\%, tomamos en consideración el estudio realizado por Sierra AS; et al, 2007, (6) en el cual se realizó 60 bloqueos anestésicos mandibulares en pacientes sometidos a la enucleación quirúrgica de terceros molares inferiores impactados, para lo cual se utilizó dos anestésicos locales: lidocaína al 2\% con epinefrina 1:100000 y articaína al 4\% con epinefrina 1:100000, de igual forma en el estudio de Ribeiro AdO; et al, 2011, (7) se tomó una muestra de forma aleatoria en la cual se aplicaron 30 bloqueos mandibulares con lidocaína 2\% y 30 bloqueos mandibulares con articaína 4\% para realizar la enucleación de órganos dentales con enfermedad periodontal crónica.

Cabe recalcar que los bloqueos mandibulares en este estudio fueron realizados por un solo operador el cual ha culminado la malla curricular de la carrera de Odontología en la Universidad Central del Ecuador, todos los bloqueos fueron supervisados por los residentes del postgrado de cirugía oral de la Facultad de Odontología de la U.C.E., con la finalidad de tener una mayor veracidad de los resultados obtenidos. 
En cuanto a la metodología utilizada en la presente investigación, tomamos en consideración los parámetros de eficacia de las soluciones anestésicas mediante el registro del tiempo de latencia, la necesidad de re-anestesiar y el nivel de control del dolor, estas se llevaron a cabo de manera meticulosa, con la finalidad de obtener datos precisos y un margen de error mínimo, siguiendo la metodología utilizada en los estudios presentados. $(6,7,8)$ Adicional a estas variables decidimos utilizar otro parámetro que permitió determinar la profundidad anestésica, como lo describe Lin NP, 2008, (9) en su publicación en la cual se indica la utilización de un testador eléctrico de pulpa con la finalidad de evaluar la intensidad anestésica.

El periodo de latencia de las soluciones anestésicas depende de algunos factores, como son las propiedades farmacológicas que tienen los anestésicos utilizados, su liposolubilidad, su nivel de afinidad a las proteínas y su capacidad de difusión en los tejidos, por otra parte el pKa de las soluciones influye directamente en el periodo de acción de los anestésicos; las soluciones anestésicas que presentan menores valores de pKa tendrán un tiempo de latencia corto; (4) acorde a esta afirmación en teoría la articaína $4 \%$ pka 7.8, presenta un menor tiempo de latencia que la lidocaína $2 \%$ pka 7.9. En el presente estudio se obtuvo como resultado un menor tiempo de latencia a favor de la articaína en promedio 94.37 segundos, en comparación con lidocaína al 2\% 162.60 segundos, por su parte Sierra-Rebolledo A, (6) en su estudio obtuvo un promedio de latencia para articaína 56 segundos y lidocaína 2\% 75 segundos, aunque la articaína presenta un mejor tiempo de latencia la diferencia entre ambas soluciones no tiene una relevancia estadística en este estudio; (8) obtuvieron como resultado en su estudio un tiempo de latencia de articaína 4\% 2.04 minutos y para lidocaína 3.23 minutos. 
Diversos factores como una mayor liposolubilidad, mayor grado de unión a proteínas, mayor concentración de anestésico local y volumen de anestésico influyen directamente en la determinación de la potencia de un anestésico, (2) bajo estos parámetros la articaína presenta, en comparación con otras soluciones anestésicas, un mejor nivel de liposolubilidad, una amplia afinidad en la fijación a proteínas, un alto nivel de difusión en los tejidos duros y blandos, una mayor concentración de anestésico en solución, esto debido a su estructura química, que es similar a la de todos los anestésicos de tipo amida a diferencia de su enlace éster y un anillo aromático tiofeno que le confiere mejores cualidades. (4) En nuestro estudio evaluamos la eficacia anestésica comparando el control del dolor transquirúrgico y la necesidad de re-anestesiar en la enucleación de órganos dentales premolares o molares inferiores; para evaluar el dolor transquirúrgico utilizamos una escala visual del dolor, en la cual obtuvimos que un $66.7 \%$ de pacientes tratados bajo anestesia con articaína refirieron no haber presentado dolor, frente a un menor porcentaje de pacientes que no presentaron dolor tratados con lidocaína 36.7\%; pese a coincidir en muchos aspectos nuestros resultados se contraponen con los obtenidos por Sierra A, (6) en los que tanto articaína $4 \%$ y lidocaína $2 \%$ presentaron similares características en el control del dolor en la extracción quirúrgica de terceros molares impactados. En contraparte la investigación realizada por Ribeiro AdO; et al, (7) reveló un mejor control del dolor por parte de la articaína $4 \%$ en la extracción de molares comprometidos por enfermedades periodontales crónicas, en total un 50\% de los casos tratados con articaína $4 \%$ refirió no haber sentido dolor frente a un $30 \%$ de los casos tratados con lidocaína $2 \%$ que refirieron no haber experimentado dolor, estos datos son similares a los obtenidos en nuestra investigación. 
En cuanto a la necesidad de usar un mayor volumen de anestésico, en nuestro estudio la lidocaína 2\% necesitó en un mayor porcentaje una segunda dosis para alcanzar el efecto anestésico en el bloqueo mandibular, $27 \%$ de los casos necesitaron ser re-anestesiados, lo que representa una tasa de éxito de $73 \%$, frente a la articaína que en un menor porcentaje de casos tratados $10 \%$ de los casos tratados necesitaron ser re-anestesiados, reflejando una tasa de éxito de 90\%; resultados similares con los presentados por Sierra A, (6) en su publicación la articaína 4\% presenta una tasa de éxito de $76.7 \%$ de los casos tratados, en comparación con la lidocaína 2\% que presentó una tasa de éxito de $62.5 \%$.

Los vitalómetros son adecuados para evaluar la profundidad obtenida en un bloqueo anestésico en dientes sanos sin inflamación pulpar o con leves inflamaciones pulpares, la utilización de estos instrumentos constituye una prueba objetiva para determinar el efecto anestésico alcanzado, posterior a la colocación de las soluciones anestésicas. (9) En nuestra investigación obtuvimos mediante prueba con vitalómetro, que se realizó en un diente sano del cuadrante anestesiado, de la cual obtuvimos como resultados que la articaína 4\%necesitó una mayor cantidad de impulso eléctrico 24.23, en relación a lidocaína $2 \%$ que necesito 16.9 para presentar respuesta de los dientes al estímulo.

Se considera entonces, oportuno sugerir la realización de otros estudios comparativos in vivo para determinar el efecto anestésico, aplicados a otros procedimientos odontológicos y en diferentes técnicas de anestesia. Los resultados encontrados en este estudio y la infinidad de reportes científicos que los respaldan, permiten afirmar que la hipótesis planteada puede ser considerada como verdadera, esto en relación a que la articaína $4 \%$ proporciona un mejor efecto anestésico comparada con lidocaína $2 \%$. 


\section{Conclusiones.}

Se determinó que la articaína 4\% con epinefrina 1:100000 (Septanest), tiene un menor tiempo de latencia en comparación con lidocaína 2\% con epinefrina 1:100000 (Lignospan Standard).

Bajo la prueba con vitalómetro se demostró que la articaína 4\% con epinefrina 1:100000 (Septanest), proporciona una mayor profundidad anestésica en comparación con lidocaína 2\% con epinefrina 1:100000 (Lignospan Standard).

Bajo la Escala Facial del Dolor la articaína 4\% con epinefrina 1:100000 (Septanest), brindo un mejor control del dolor transquirúrgico, con un mayor porcentaje de pacientes que refirieron “Ausencia de Dolor”, en comparación con lidocaína 2\% con epinefrina 1:100000 (Lignospan Standard).

La lidocaína 2\% con epinefrina 1:100000 (Lignospan Standard), necesitó en un mayor porcentaje de los casos tratados una segunda dosis para alcanzar el efecto anestésico deseado, en comparación con la articaína 4\% con epinefrina 1:100000 (Septanest).

\section{Bibliografía.}

1. Vásquez E, Ramos R, Villalobos E. Estudio comparativo de la efectividad de dos anestésicos locales. Medicina Oral. 2003; 5(3): p. 79-83.

2. Macouzet C. Anestesia local en odontología. 2nd ed. México: Editorial Manual Moderno; 2005.

3. Martínez-Martínez A. Anestesia Bucal, Guía Práctica. 2nd ed. Bogoá: Panamericana; 2009.

4. Malamed S. Manual de Anestesia Local. 5th ed. Madrid: Elsevier; 2006.

5. Ensaldo-Carrasco E, Ensaldo-Carrasco E, Rivas-Muñoz R, Ensaldo-Fuentes E, Adler-Schiller S. Estudio clínico comparativo entre articaína. ADM. 2003 dic; 60(6): p. 212-218. 
6. Sierra-Rebolledo A, Delgado-Molina E, Berini-Aytés L, Gay-Escoda C. Estudio comparativo sobre la eficacia anestésica entre la Articaína al $4 \%$ y la Lidocaína al $2 \%$ en el bloqueo del nervio alveolar inferior durante la extracción quirúrgica de los terceros molares inferiores incluidos. Medicina oral, patología oral y cirugía bucal. Ed. española. 2007; 12(2): p. 101-106.

7. Ribeiro A, Silveira C, Cruz G, Souza L. Comparação da Eficácia Anestésica da Articaína e da Lidocaína em Extrações de Dentes com Periodontite Crônica Avançada. Pesq Bras Odontoped Clin Integr. 2011; 11(3): p. 329-333.

8. Vimal K, Supreet, Rupinder K. Comparative evaluation of onset and duration of anesthesia of $4 \%$ articaine versus 2\% lidocaine with epinephrine 1:1,00,000 during exodontia. Indian Journal of Comprehensive Dental Care (IJCDC). 2011; 1(1): p. 19.

9. Lin N. Electric pulp testing: a review. International Endodontic Journal. 2008; 41(5): p. 365-374. 